\title{
Acta
Biochimica
Polonica
}

Vol. 40 No. 4/1993

QUARTERLY

\section{Discrimination between chilling-sensitive and chilling-resistant plants based on measurements of free fatty acid accumulation and inactivation of oxygen evolution in aged chloroplasts ${ }^{* * * *}$}

\author{
Violetta Saczyńska, Joanna Kargul and Zbigniew Kaniuga \\ Institute of Biochemistry, University of Warsaw, Al. Żwirki i Wigury 93, 02-089 Warsaw, Poland
}

Received 2 July, 1993

\begin{abstract}
The effect of aging of isolated chloroplasts of two chilling-sensitive (CS) and three chilling-resistant (CR) plants on the inactivation of oxygen evolution and accumulation of free fatty acids (FFA) was studied at $30^{\circ} \mathrm{C}, \mathrm{pH} 5.5$ or 7.0 , in the absence or presence of either sorbitol or $\mathrm{NaCl}$.

Considerable accumulation of FFA in aged chloroplasts of CS plants: bean and maize line F7-RpIII was accompanied by a marked inactivation of oxygen evolution. This relation was not, however, found in chloroplasts of CR species: pea, wheat and maize line EP1-RpI, in which the accumulation of FFA upon aging was very low whereas the decline of the rate of oxygen evolution was pronounced.

In contrast to changes observed at $\mathrm{pH} 5.5$, the inactivation of oxygen evolution in chloroplasts of $\mathrm{CR}$ species aged at $\mathrm{pH} 7.0$ was dependent on the composition of the medium, especially in wheat chloroplasts. Thus, for the evaluation of chilling sensitivity based on the measurements of oxygen evolution activity solely, either aging of chloroplasts at pH 5.5 or possibly at pH 7.0 with $\mathrm{NaCl}$ included into the incubation medium may be recommended.

It is concluded that determination of both the extent of FFA accumulation and inactivation of oxygen evolution in aged chloroplasts might be applied as chilling tolerance indexes.
\end{abstract}

In the first concept of chilling sensitivity of plants the degree of fatty acid unsaturation in membrane lipids was considered to be a critical factor contributing to the ability of plants to survive chilling temperatures [1]. It was also postulated that the content of phosphatidylglycerol molecules containing two high melting point fatty acids $\left(16: 0+\right.$ trans $\left.-\Delta^{3}-16: 1\right)$ influences the temperature at which phase transition takes place in bulk membrane lipids [2 - 4]. Both hypotheses are well known but did not avoid several critical comments concerning the participation of unsaturated fatty acids [5] and of phosphatidylglycerol molecular species in phase transition of membrane lipids $[6,7]$.

On the other hand, our studies indicated that there is another characteristic feature of chilling-sensitive plants, namely a higher activity of chloroplast lipid acyl hydrolase (galactolipase EC 3.1.1.26) (LAH) in chilling-sensitive (CS) than in chilling-resistant (CR) species [8 - 12]. This property of the enzyme is responsible for few consecutive events such as: more extensive accumulation of free fatty acids (FFA) in chlo-

*This work is a part of the project supported by the State Committe for Scientific Research grant No. 406069101.

**This is the 30th paper of the series: "Photosynthetic apparatus of chilling-sensitive plants".

${ }^{1}$ Abbreviations: Chl, chlorophyll; $C S$, chilling-sensitive; $C R$, chilling-resistant; FFA, free fatty acids; LAH, lipid acyl hydrolase; MGDG, monogalactosyldiacylglycerol. 
roplasts upon chilling [8 - 12], the depletion of functionally active $\mathrm{Mn}[13-15]$, the inactivation of oxygen evolution [10 - 13, $15-18]$ and the damage of chloroplast structure $[19,20]$. In CR species such changes are not observed [19]. Thus, it was concluded that chilling-induced degradation of thylakoid lipids by LAH in CS plants affects the composition and therefore the fluidity of membranes and their physical properties responsible for phase transition.

All these changes induced by chilling stress applied to detached leaves seem to be analogous to those occurring in aged chloroplasts [ 21 - 23]. Therefore in this work we assume that extent of FFA accumulation and inactivation of oxygen evolution in isolated chloroplasts upon aging could serve as an index of susceptibility of plants to chilling.

Our experiments on the effect of $\mathrm{pH}$ and detergents on FFA accumulation in aged chloroplasts of CS plants demonstrated that this process is influenced by $\mathrm{pH}$ (Saczyńska et al., unpublished). Moreover, it was reported [24] that the rate of fatty acid release in spinach chloroplasts isolated and aged in the medium containing $\mathrm{NaCl}$ was higher than in those isolated and aged in mannitol or sucrose media. On the other hand, it might be presumed that elevated temperature, $\mathrm{pH}$ and the composition of the incubation mixture could affect both oxygen evolution and galactolipase activity. Thus, the aim of this work was to establish conditions of chloroplast aging the most suitable for discrimination between $C S$ and $C R$ plants.

\section{MATERIAL AND METHODS}

Plant material and chloroplast isolation. In the present studies two species of CS plants bean (cv. Eureka) and maize (line F7-RpIII) and three species of CR plants - pea (cv. Nike), wheat (cv. Parada) and maize (line EP1-RpI) were used. Seeds were soaked in aerated water for a few hours and allowed to germinate on moist paper towels in darkness. Then seedlings were grown at about $24^{\circ} \mathrm{C}$ (day) and $18^{\circ} \mathrm{C}$ (night) under $16 \mathrm{~h}$ photoperiod. Light, approx. $80 \mu \mathrm{mol} \times \mathrm{m}^{-2} \times \mathrm{s}^{-1}$, was provided by the "day light" fluorescent tubes (Polam, Poland). Seedlings were watered with Knopp's solution and distilled water, and leaves of the 2-3-week-old plants were harvested. Chloroplasts were isolated and the chlorophyll content was estimated as described previously [12].

Aging of chloroplasts. The suspension of freshly isolated chloroplasts was diluted with the isolation buffer to contain $5 \mathrm{mg} \mathrm{Chl}$ per $\mathrm{ml}$. The aliquots of $0.20 \mathrm{ml}$ of the diluted chloroplast suspension was added to $2.30 \mathrm{ml}$ of the incubation mixture, of $\mathrm{pH} 5.5$ or 7.0 , containing alternatively: (a) $0.109 \mathrm{M}$ Tris/maleate buffer, (b) $0.109 \mathrm{M}$ Tris/maleate buffer plus $0.24 \mathrm{M}$ sorbitol, and (c) $0.109 \mathrm{M}$ Tris/maleate buffer plus $0.12 \mathrm{M} \mathrm{NaCl}$. The calculated osmomolarity of the media containing sorbitol or $\mathrm{NaCl}$ was the same as of the buffers used for isolation of chloroplasts.

Aging of chloroplasts was carried out at $30^{\circ} \mathrm{C}$ during $1 \mathrm{~h}$ in the incubation media mentioned above and the samples were withdrawn after 20,40 and $60 \mathrm{~min}$ of incubation for the determination of FFA content and oxygen evolution. Susceptibility of isolated chloroplasts to aging was studied in $2-4$ experiments for each species examined.

Isolation and determination of FFA. In both control and incubated samples of chloroplasts (equivalent to $1 \mathrm{mg} \mathrm{Chl}$ ) the lipid hydrolysis was stopped with acidic ethanol, and FFA were extracted with hexane. The efficiency of fatty acid extraction was estimated using $\left[{ }^{14} \mathrm{C}\right]$ palmitic acid as an internal standard. The FFA content was determined by the modified colorimetric method with 1,5-diphenylcarbazide [25] (to be published elsewhere). The comparison of the results obtained by this method with those obtained by GLC demonstrated usefulness of the colorimetric method in comparative studies.

Determination of oxygen evolution. Oxygen evolution was followed polarographically with Clark type electrode (Yellow Springs Instrument Co., U.S.A.). The reaction mixture contained: $20 \mathrm{mM}$ Tris/maleate buffer, $\mathrm{pH} 7.0,0.4$ $M$ sucrose, $15 \mathrm{mM} \mathrm{NaCl}, 5 \mathrm{mM} \mathrm{MgCl}, 0.4 \mathrm{mM}$ phenyl-p-benzoquinone, $3 \mathrm{mM}$ methylamine and chloroplasts $\left(13-18 \mu \mathrm{g} \mathrm{Chl} \times \mathrm{ml}^{-1}\right)$ in a 2.54 $\mathrm{ml}$ volume. The reaction was carried out at $25^{\circ} \mathrm{C}$ under saturating light (about $900 \mu \mathrm{mol} \times$ $\mathrm{m}^{-2} \times \mathrm{s}^{-1}$ ). Recordings of electrode signals and calculations of the oxygen evolution rate were made automatically using a programme for IBM-PC computer with ADDA Card. 
Presentation of results. The amount of fatty acids released and the extent of inactivation of oxygen evolution during incubation are expressed in $\mu \mathrm{mol}$ FFA and oxygen, recalculated per $\mathrm{mg} \mathrm{Chl}$ and per $\mathrm{mg} \mathrm{Chl} \mathrm{per} \mathrm{h,} \mathrm{respectively.}$ The data presented are means \pm SE of $2-4$ experiments.

\section{RESULTS}

\section{Inactivation of oxygen evolution}

The inactivation of oxygen evolution following incubation of chloroplasts of CS and CR plants at $\mathrm{pH} 5.5$ or 7.0 is presented in Figs. 1 - 3 (bottom). As can be seen, there are significant differences between CS and CR plants in respect of changes in oxygen evolution upon incubation. These changes were much higher in chloroplasts isolated from CS bean and maize line F7-RpIII than in those of CR plants. In chloroplasts of CS plants, the inactivation of oxygen evolution was more extensive at $\mathrm{pH} 5.5$ than at 7.0 and was the most rapid during the first 20 min of incubation. On the other hand, in chloroplasts of $C R$ plants a gradual decrease of oxygen evolution was observed during 60 min of incubation. Moreover, the presence of sorbitol or $\mathrm{NaCl}$ in the Tris/maleate incubation mixture diminished inactivation of oxygen evolution in chloroplasts of CR plants aged at pH 7.0 (Figs. 1B - 3B).

\section{Accumulation of FFA}

In general, inactivation of oxygen evolution upon aging of chloroplasts is related to the extent of simultanously released fatty acids as it was previously demonstrated with chilled leaves [10, 12, 18, 28]. As shown in Figs. 1 - 3 (upper parts) accumulation of FFA was markedly higher in chloroplasts of CS species: bean and maize line F7-RpIII incubated in all three media, than in those of CR species: pea, wheat and maize line EP1-RpI. The release of fatty acids in chloroplasts of CS plants was again the most rapid within the first 20 min of incubation. It was faster and greater at $\mathrm{pH} 5.5$ than at 7.0 but at given $\mathrm{pH}$ was not influenced by the presence of $\mathrm{NaCl}$ or sorbitol in the reaction mixture. In contrast, in chloroplasts of $C R$ plants the extent of fatty acid release during aging was extremely low at both $\mathrm{pH} 5.5$ and 7.0 and in all three media applied.
Inactivation of oxygen evolution versus FFA accumulation

In chloroplasts of CS plants: bean and maize line F7-RpIII the extent of both the inactivation of oxygen evolution and accumulation of FFA during aging depended on $\mathrm{pH}$ of the assay mixture. Greater inactivation of oxygen evolution in chloroplasts aged at $\mathrm{pH} 5.5$ than at 7.0 was accompanied by more extensive accumulation of FFA (Figs. 1 - 3). Both kinds of responses were progressive with time.

In chloroplasts of CR plants there was no relation between oxygen evolution and the amount of fatty acids released either at $\mathrm{pH} 5.5$ or 7.0 (Figs. $1-3$, upper parts). The accumulation of FFA in chloroplasts of all three species was very low, while the inactivation of oxygen was pronounced. This suggests that other factor(s) are involved in the inactivation of photochemical activity. The degradation of galactolipids can be excluded due to the insignificant increase of FFA level, independently of $\mathrm{pH}$ at aging.

\section{DISCUSSION}

The acyl lipid composition of leaves and chloroplasts of CS and CR plants does not differ essentially [26]. Similarly, the content of the high melting point fatty acids in phosphatidylglycerol in chloroplasts of CS and CR species is not a sufficient criterion to distinguish between these two groups of plants [6]. The content of FFA in freshly isolated chloroplasts of CR plants is generally lower than in CS ones [9] but variable in both groups of plants. Even among closely related CS cultivars the original content of FFA in chloroplasts differs greatly, e.g. by a factor of two in tomato [10], about three in cucumber [12], four in maize [18], five in melon [28] and 6.5 in red pepper [18]. Moreover the composition of FFA in the thylakoid membranes of CS plants within the same species but differing in chilling tolerance indicated some small deviations from the general pattern in the proportion of individual fatty acids $[12,27]$. However, there are no corresponding data for CR plants.

It is interesting that the high original level of FFA in chloroplasts of some red pepper cvs and maize lines remained constant during a few 


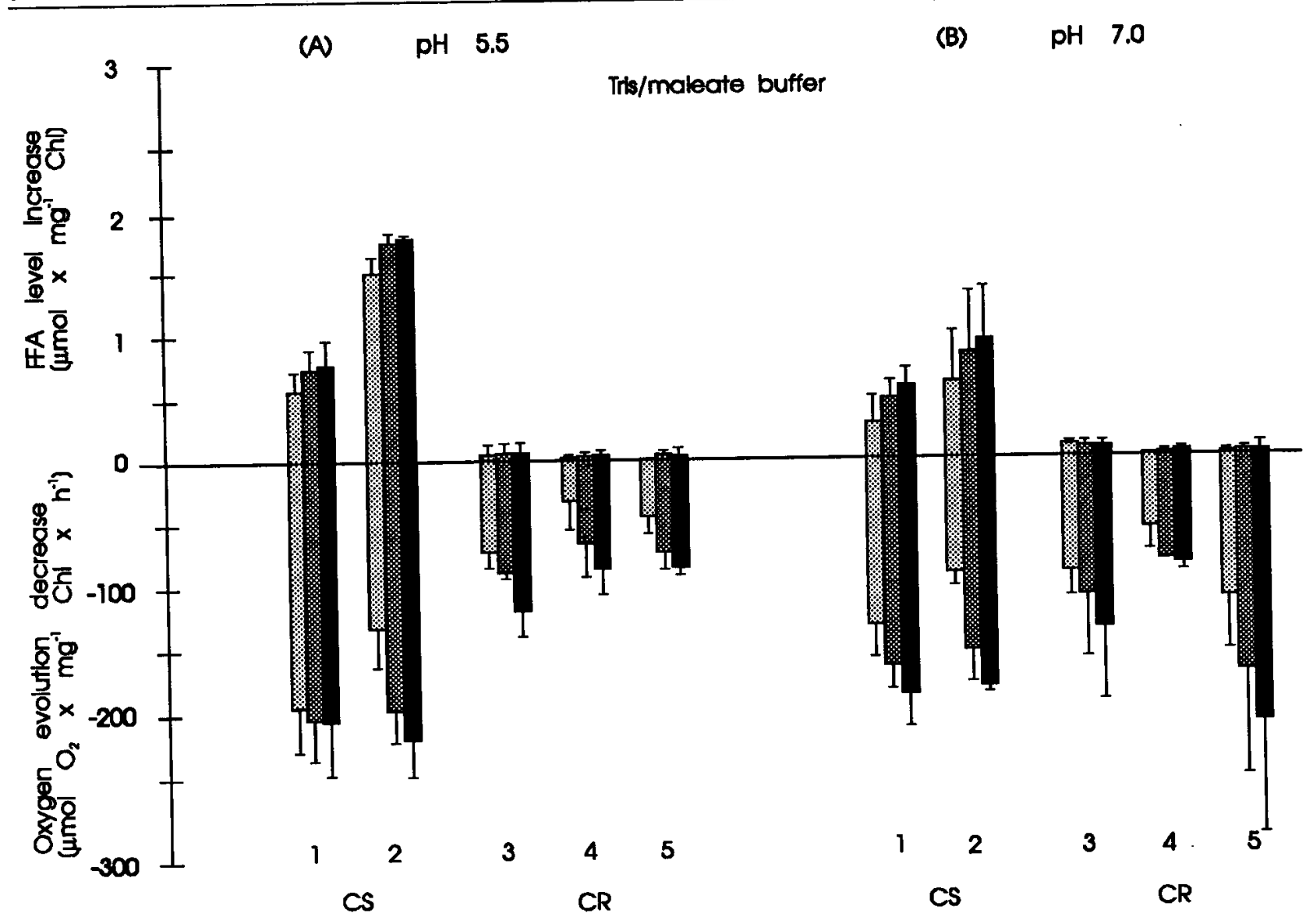

Fig. 1. Decrease of oxygen evolution and increase of FFA level during aging of isolated chloroplasts in the incubation mixture, pH $5.5(A)$ or $p H 7.0(B)$, containing Tris/maleate buffer only.

Chloroplasts of the chilling-sensitive (CS) plants: bean (1) and maize - line F7-RpIII (2) and chilling-resistant (CR) plants: maize - line EP1-RpI (3), pea (4), and wheat (5) were used. Oxygen evolution and FFA level were measured after 20 (grey bars), 40 (hatched bars) and 60 (black bars) min of chloroplast incubation at $30^{\circ} \mathrm{C}$ in Tris/maleate buffer. Bars with vertical lines represent means \pm SE of 2 to 4 experiments.

days of chilling and this level was not related to oxygen evolution [18]. Similarly, the original higher level of FFA in the chilling tolerant cucumber species than in the CS ones was not accompanied by a lower photochemical activity [12]. These findings are consistent with those shown with purified and non-purified chloroplasts under aging conditions [29] as no quantitative correlation was established between the total amount of FFA present in chloroplasts and the degree of inhibition of electron transport activity. In non-purified thylakoids most of the fatty acids released on the external side of the membrane by contaminating lipolytic enzymes are not inhibitory for the electron transport in contrast to the fatty acids released in the purified membranes by intrinsic lipase(s). Thus, an increase in FFA during chilling stress depends mainly on the thylakoid intrinsic galactolipase activity of the plant, and stress conditions $[8,10-13,15,16,18,19]$. All these observations may imply that, in chloroplasts, two pools of FFA are present. The first, of variable amount depending on the plant species and the isolation procedure, exists in freshly isolated chloroplasts and does not affect

Fig. 2. Decrease of oxygen evolution and increase of FFA level during aging of isolated chloroplasts in incubation mixture, pH $5.5(A)$ or $\mathrm{pH} 7.0(B)$, containing Tris/maleate buffer and sorbitol. For explanations see legend to Fig. 1.

Fig. 3. Decrease of oxygen evolution and increase of FFA level during aging of isolated chloroplasts in incubation mixture, pH $5.5(A)$ or $p H 7.0$ (B), containing Tris/maleate buffer and $\mathrm{NaCl}$. For explanations see legend to Fig. 1. 


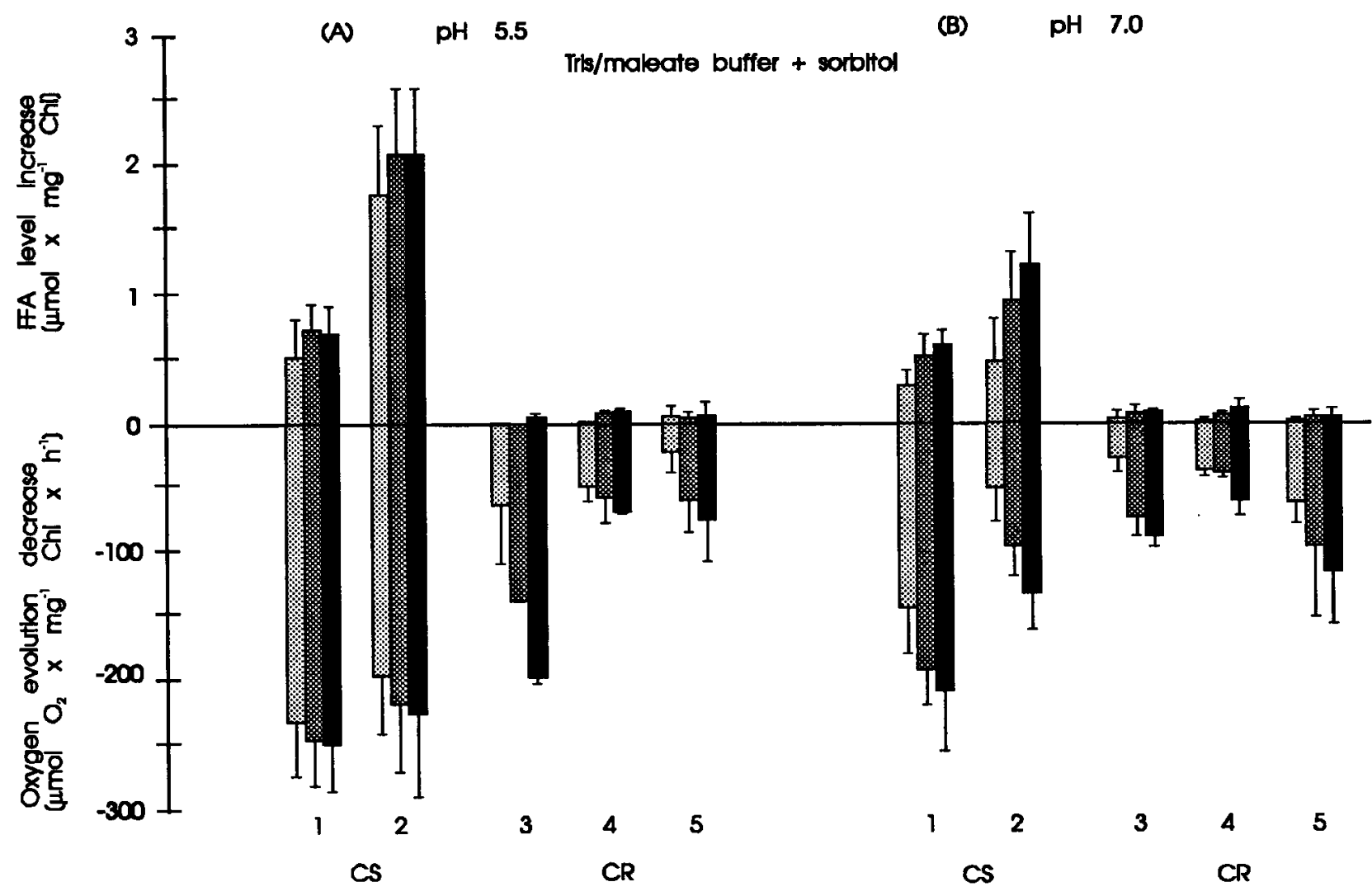

Fig. 2

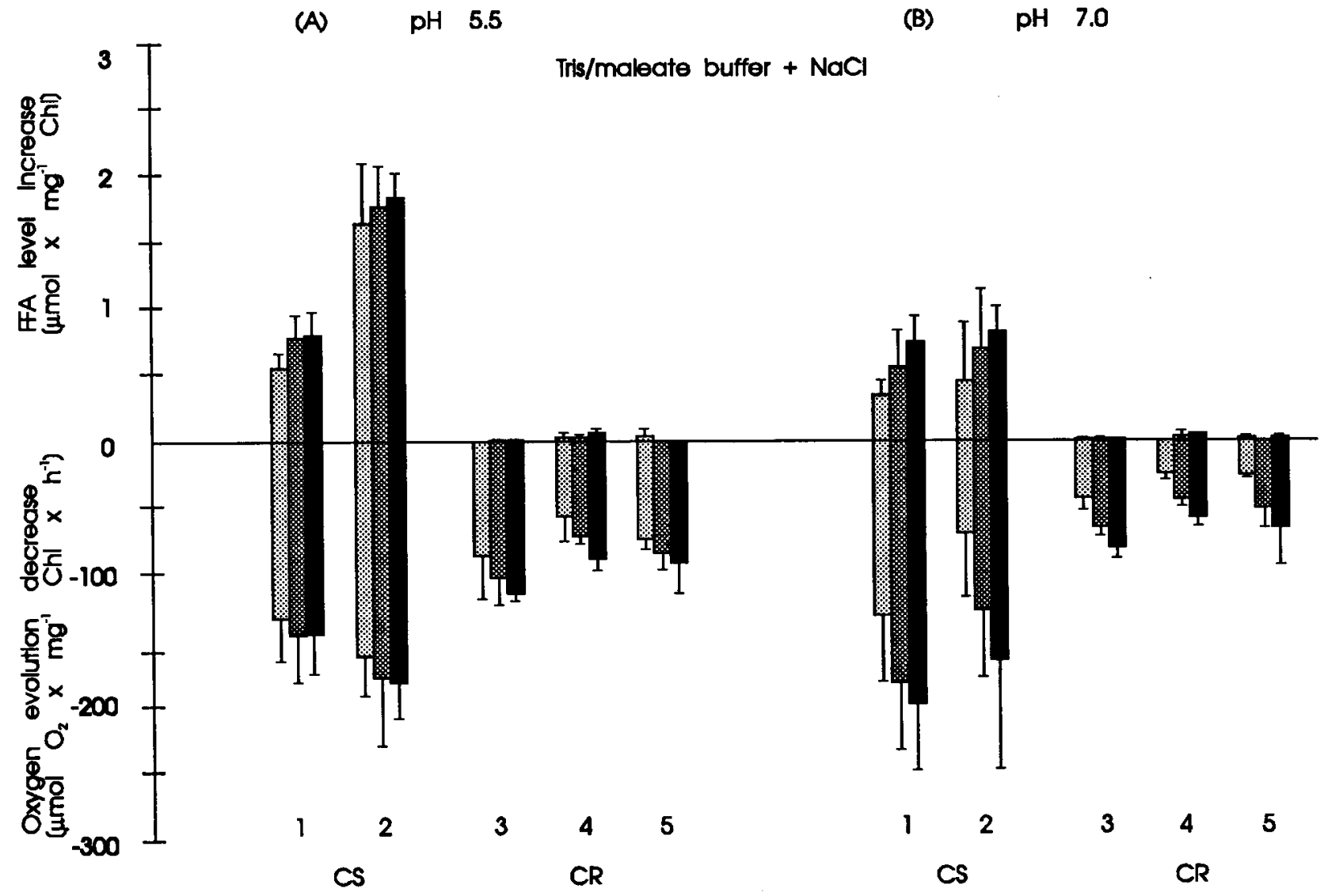

Fig. 3 
photochemical activity $[12,18,29]$. The second, generated during chilling stress [12] or aging of chloroplasts of CS plants (Figs. 1 - 3) is responsible for the inactivation of oxygen evolution and results from the enhanced galactolipase activity characteristic of CS plants. In contrast, in chloroplasts of $C R$ plants, the release of fatty acids is low even upon incubation at $30^{\circ} \mathrm{C}$, due to very low galactolipase activity (Figs. $1-3$ ).

There are data indicating that the level of endogenously generated FFA is about ten times more effective in the inhibition of chloroplast photochemical activity than exogenous linolenic acid [21,23]. This is probably due to the differences in the site and mode of action of endogenous and exogenous FFA.

Losses of functional activity in chloroplasts during aging have often been attributed to the accumulation of fatty acids released from membrane lipids by galactolipase $[22,30]$. The lack of such correlation was, however, found only in chloroplasts of CR plants: pea, wheat and maize line EP1-RpI (Figs. 1 -3) in which the release of fatty acids upon incubation was very low while the inactivation of oxygen evolution was appreciable. These results indicate a very low activity of galactolipase and that inactivation of oxygen evolution is governed by the factors other than FFA.

In conclusion, in chloroplasts of CR plants such as lettuce and spinach [29], horse bean and spinach [23], pea, wheat and chilling tolerant maize (Figs. 1 - 3) no direct correlation exists between endogenous fatty acid release and the loss of chloroplast function upon aging.

In chloroplasts of CS plants the inactivation of oxygen evolution was accompanied by considerable accumulation of FFA (Figs. 1 - 3). Moreover, the extent and rate of changes in both oxygen evolution and FFA level were found to be $\mathrm{pH}$-dependent (Figs. 1 - 3). Thus our results indicate that at least two factors are involved in the deterioration of the oxygen evolution activity in aged chloroplasts of CS plants i.e. degradation of membrane lipids by galactolipase and accumulation of FFA. These events seem to be responsible for greater inactivation of oxygen evolution in aged chloroplasts of CS plants in comparison to those of CR ones. Similar conclusions were obtained with detached leaves under chilling conditions $[10,12,18,28]$.

The data presented suggest that the extent of FFA accumulation and inactivation of oxygen evolution in chloroplasts upon chilling of leaves or aging of chloroplasts are characteristic parameters discriminating between CS and CR plants and can be applied to both assay systems.

We wish to thank Mr Zbigniew Kurczych, Director of the Plant Breeding Station in Kobierzyce, for providing maize seeds of chillingsensitive line F7-Rp-III and chilling-resistant line EP1-RpI. We are grateful to Ewa Miśkiewicz and Marek Rzepecki for preparation of Figures.

\section{REFERENCES}

1. Lyons, J.M. (1973) Chilling injury in plants. Annu. Rev. Plant Physiol. 24, 445 - 466.

2. Murata, N. (1983) Molecular species composition of phosphatidylglycerols from chilling-sensitive and chilling-resistant plants. Plant Cell Physiol. 24, 81 - 86.

3. Raison, J.K. \&Wright, L.C. (1983) Thermal phase transition in the polar lipids in plant membranes. Biochim. Biophys. Acta 371, 69 - 78.

4. Orr, G.R. \& Raison, J.K. (1987) Compositional and thermal properties of thylakoid polar lipids of Nerium oleander L. in relation to chilling sensitivity. Plant Physiol. 84, 88 - 92.

5. Quinn, P.J. \& Williams, W.P. (1983) The structural role of lipids in photosynthetic membranes. Biochim. Biophys. Acta 737, 223-266.

6. Bishop, D.G. (1986) Chilling sensitivity in higher plants: the role of phosphatidylglycerol. Plant Cell Environ. 9,613-616.

7. Pike, C.S., Norman, H.A., Kemmerer, E.C., Wessner, D.R., Greenberg, C.M., Kaplan, L.J., Brodsky, N.M. \& Ellis, A.A. (1990) Effects of acclimation to low temperature and to water stress on photosynthesis and on physical and chemical properties of lipids from thylakoids of cucumber and cotton. Plant Sci. 68, 189 - 196.

8. Kaniuga, Z. \& Gemel, J. (1984) Galactolipase activity and free fatty acid levels in chloroplasts. Novel approach to characteristics of chilling sensitivity of plants. FEBS Lett. 171, 55 - 58.

9. Gemel, J. \& Kaniuga, Z. (1987) Comparison of galactolipase activity and free fatty acid levels in chloroplasts of chill-sensitive and chill-resistant plants. Eur. J. Biochem. 166, 229 233.

10. Gemel, J., Sączyńska, V. \& Kaniuga, Z. (1988) Galactolipase activity and free fatty acid levels 
in chloroplasts of domestic and wild tomatoes with different chilling tolerance. Physiol. Plant. 74, 509 - 514 .

11. Gemel, J., Cieśla, E. \& Kaniuga, Z. (1989) Different response of two Zea mays inbreds to chilling stress measured by chloroplast galactolipase activity and free fatty acid levels. Acta Physiol. Plant. 11, 3 - 11.

12. Saczyńska, V., Gemel., J. \& Kaniuga, Z. (1993) Chilling susceptibility of Cucumis sativus species. Phytochemistry 33, 61 - 67.

13. Kaniuga, Z., Zabek, J. \& Sochanowicz, B. (1978) Photosynthetic apparatus of chilling sensitive plants. III. Contribution of loosely bound manganese to the mechanism of reversible inactivation of Hill reaction activity following cold and dark storage of leaves. Planta 144, 49 56.

14. Kaniuga, Z., Gemel, J. \& Zabłocka, B. (1986) Fatty acid-induced release of manganese from chloroplasts. Biochim. Biophys. Acta 850, 473 482.

15. Garstka, M. \& Kaniuga, Z. (1991) Reversal by light of deletorious effects of chilling on oxygen evolution, manganese and free fatty acid content in tomato thylakoids is not accompanied by restoration of the original membrane conformation. Physiol. Plant. 82, 292 - 298.

16. Kaniuga, Z., Sochanowicz, B., Zabek, J. \& Krzystyniak, K. (1978) Photosynthetic apparatus of chilling sensitive plants. I. Reactivation of Hill reaction activity inhibited on the cold and dark storage of detached leaves and intact plants. Planta 140, 121 - 128.

17. Kaniuga, Z. \& Michalski, W.P. (1978) Photosynthetic apparatus of chilling sensitive plants. II. Changes in free fatty acid composition and photoperoxidation in chloroplasts following cold storage and illumination of leaves in relation to Hill reaction activity. Planta 140, 129 $-136$.

18. Sączyńska, V., Gemel, J. \& Kaniuga, Z. (1990) Effect of chilling of Zea mays L. and Capsicum annuum $\mathrm{L}$. leaves on inactivation of oxygen evolution and content of free fatty acids in chloroplasts. Acta Physiol. Plant. 12, 239 - 245.

19. Gemel, J. Golinowski, W. \& Kaniuga, Z. (1986) Low-temperature induced changes in chloroplast ultrastructure in relation to changes of Hill reaction activity, manganese and free fatty acid levels in chloroplasts of chilling-sensitive and chilling resistant plants. Acta Physiol. Plant. 8, 135 - 143.

20. Wise, R.T. \& Naylor, A.W. (1987) Chilling enhanced photoperoxidation. The peroxidative destruction of lipids during chilling injury to photosynthesis and ultrastructure. Plant Physiol. 83, 272 - 277.

21. Constantynopoulos, G. \& Kenyon, Ch.N. (1968) Release of free fatty acids and loss of Hill activity by aging spinach chloroplasts. Plant Physiol. 43, $531-536$.

22. Siegenthaler, P.-A. \& Rawyler, A. (1977) Aging of the photosynthetic apparatus. V. Change in $\mathrm{pH}$ dependence of electron transport and relationship to endogenous free fatty acids. Plant Sci. Lett. 9, 265 - 273.

23. Pencival, M.P., Williams, W.P., Chapman, D.J. \& Quinn, P.J. (1980) Loss of Hill activity in isolated chloroplasts is not directly related to free fatty acid release during aging. Plant Sci. Lett. 19, 47 54.

24. Wintermans, J.F.G.M., Helmsing, P.J., Polman, B.J.J., van Gisbergen, J. \& Collard, J. (1969) Galactolipid transformations and photochemical activities of spinach chloroplasts. Biochim. Biophys. Acta 189, 95 - 105.

25. Nixon, N. \& Chan, S.H. (1979) A simple and sensitive colorimetric method for the determination of long-chain free fatty acids in subcellular organelles. Anal. Biochem. 97, 403 409.

26. Harwood, J.L. (1980) Plant acyl lipids structure, distribution, and analysis; in The Biochemistry of Plants. Lipids: Structure and Function (Stumpf, P.K., ed.) vol. 4, pp. 1 - 55, Academic Press, New York.

27. Gemel, J., Sączyńska, V. \& Kaniuga, Z. (1989) Composition of non-esterified fatty acids in chloroplasts of closely related chill-sensitive plants. Phytochemistry 28, 1813 - 1816.

28. Gemel, J. \& Kaniuga, Z. (1989) Galactolipase activity and free fatty acids in chloroplasts as indicators of chilling sensitivity of closely related plant species; in Techniques and New Developments in Photosynthesis Research (Barber, J. \& Malkin, R., eds.) pp. 597-600, Plenum Press, New York.

29. Siegenthaler, P.-A., Rawyler, A. \& Henry, L.E.A. (1981) A new type correlation between changes in lipid composition and loss of electron transport activities during aging in vitro; in Photosynthesis. II. Electron Transport and Photophosphorylation (Akoyunoglou, G., ed.) pp. 167 - 174, Balaban International Science Services, Philadelphia.

30. Shaw, A.B., Anderson, M.M. \& McCarty, R.E. (1976) Role of galactolipids in spinach chloroplasts lamellar membranes. II. Effects of galactolipid depletion on phosphorylation and electron flow. Plant Physiol. 57, 724 - 729. 